\title{
ON THE MEAN MODULUS OF TRIGONOMETRIC POLYNOMIALS WHOSE COEFFICIENTS HAVE RANDOM SIGNS
}

\section{S. UCHIYAMA}

1. Introduction. Let $\left(m_{k}\right)$ be an infinite sequence of distinct integers. We denote by $s_{n}(t, x)(0<t<1,0 \leqq x<1)$ the $n$th partial sum of the trigonometric series

$$
\sum_{k=1}^{\infty} \phi_{k}(t) c_{k} e\left(m_{k} x\right)
$$

where $\left(\phi_{k}(t)\right)$ is the system of Rademacher's functions, i.e.

$$
\phi_{k}(t)=\operatorname{sign} \sin 2^{k} \pi t \quad(k=1,2, \cdots),
$$

and $e(x)=e^{2 \pi x}$. The coefficients $c_{k}$ may be complex numbers.

R. Salem and A. Zygmund [2] have investigated in detail the order of magnitude of the maximum modulus of $s_{n}(t, x)$,

$$
\max _{0 \leqq x<1}\left|s_{n}(t, x)\right| \text {, }
$$

for the special case of $\left(m_{k}\right)=(k)$, the sequence of positive integers. The purpose of this note is to present some results concerning the order of magnitude of the mean modulus

$$
\int_{0}^{1}\left|s_{n}(t, x)\right| d x,
$$

where the sequence $\left(m_{k}\right)$ may be arbitrary.

It is clear that for every $t$ and every $n$

$$
\int_{0}^{1}\left|s_{n}(t, x)\right| d x \leqq R_{n}^{1 / 2},
$$

where $R_{n}=\sum_{k=1}^{n}\left|c_{k}\right|^{2}$, whatever the sequence $\left(m_{k}\right)$ may be. As to the lower bound for $\int_{0}^{1}\left|s_{n}(t, x)\right| d x$ we shall prove the following theorems:

TheOREM 1. Let $\left(m_{k}\right)$ be an arbitrary sequence of distinct integers. Then, given any $\epsilon>0$, there exists a positive constant $B_{c}$ depending only on $\epsilon$, such that except for a set of $t$ 's of measure less than $\epsilon$ we have

Received by the editors December 14, 1964. 


$$
\int_{0}^{1}\left|s_{n}(t, x)\right| d x \geqq B_{\epsilon} R_{n}^{1 / 2}
$$

for all $n \geqq 1$.

We write for the sake of brevity

$$
S_{n}=\sum\left|c_{i}\right|^{2}\left|c_{j}\right|^{2}\left|c_{k}\right|^{2}\left|c_{l}\right|^{2}
$$

the summation being extended over all indices $i, j, k, l$ with $1 \leqq i, j$, $k, l \leqq n$ such that $m_{i}+m_{j}=m_{k}+m_{l}$ (order is relevant).

THEOREM 2. Let $\left(m_{k}\right)$ be an arbitrary sequence of distinct integers. If $S_{n} / R_{n}^{4}=O\left(n^{-\alpha}\right)$ for some $\alpha>1$, then we have

$$
\liminf _{n \rightarrow \infty} R_{n}^{-1 / 2} \int_{0}^{1}\left|s_{n}(t, x)\right| d x \geqq 2^{-1 / 2}
$$

almost everywhere in $t$.

The condition imposed on $S_{n} / R_{n}^{4}$ in Theorem 2 obviously implies that $R_{n}$ tends to infinity with $n$. Note that for any sequence $\left(m_{k}\right)$ of distinct integers we have always $1 \leqq \alpha \leqq 2$, if $S_{n} / R_{n}^{4}=O\left(n^{-\alpha}\right)$; in the case of $\alpha=1$, which is excluded from Theorem 2 , one may also prove the validity of (2), assuming that the growth of $R_{n}$ as $n \rightarrow \infty$ is sufficiently regular (see $\$ 3$ below).

2. Proof of the theorems. We have

$$
\int_{0}^{1}\left|s_{n}(t, x)\right|^{2} d x=\sum_{k=1}^{n} \phi_{k}^{2}(t)\left|c_{k}\right|^{2}=R_{n}
$$

almost everywhere in $t$. Hence we obtain by Hölder's inequality

$$
\begin{aligned}
R_{n} & =\int_{0}^{1}\left|s_{n}(t, x)\right|^{2} d x \\
& \leqq\left(\int_{0}^{1}\left|s_{n}(t, x)\right| d x\right)^{2 / 8}\left(\int_{0}^{1}\left|s_{n}(t, x)\right|^{4} d x\right)^{1 / 8}
\end{aligned}
$$

for almost all $t$, where, as is readily seen,

$$
\int_{0}^{1} d t \int_{0}^{1}\left|s_{n}(t, x)\right|^{4} d x=2 R_{n}^{2}-T_{n}, \quad T_{n}=\sum_{k=1}^{n}\left|c_{k}\right|^{4}
$$

Let $E$ denote the set of $t, 0<t<1$, for which the integral 


$$
\int_{0}^{1}\left|s_{n}(t, x)\right|^{4} d x \geqq 2 R_{n}^{2}-T_{n}+A .
$$

Then it follows from (4) that the measure $m(E)$ of $E$ satisfies

$$
m(E) \leqq \frac{2 R_{n}^{2}-T_{n}}{2 R_{n}^{2}-T_{n}+A} \leqq \frac{2}{2+a}<\epsilon,
$$

if we put $A=a R_{n}^{2}$ and take $a>0$ sufficiently large. Thus, for any $t \notin E$ we have

$$
\int_{0}^{1}\left|s_{n}(t, x)\right|^{4} d x<(2+a) R_{n}^{2}
$$

and we obtain, via (3),

$$
\int_{0}^{1}\left|s_{n}(t, x)\right| d x \geqq \frac{R_{n}^{3 / 2}}{(2+a)^{1 / 2} R_{n}}=B_{a} R_{n}^{1 / 2}
$$

with $B_{\mathbf{6}}=(2+a)^{-1 / 2}$. This is (1).

Now, let us consider the integral

$$
I_{n}=\int_{0}^{1} R_{n}^{-4}\left(\int_{0}^{1}\left|s_{n}(l, x)\right|^{4} d x-2 R_{n}^{2}+T_{n}\right)^{2} d t .
$$

It is not difficult to verify that

$$
\int_{0}^{1}\left(\int_{0}^{1}\left|s_{n}(t, x)\right|^{4} d x\right)^{2} d t=\left(2 R_{n}^{2}-T_{n}\right)^{2}+O\left(S_{n}\right)
$$

By the assumption $S_{n} / R_{n}^{4}=O\left(n^{-\alpha}\right)(\alpha>1)$ and the relation (4) we thus have $I_{n}=O\left(n^{-\alpha}\right)$ and therefore $\sum_{1}^{\infty} I_{n}<\infty$. Hence

$$
\sum_{n=1}^{\infty} R_{n}^{-4}\left(\int_{0}^{1}\left|s_{n}(t, x)\right|^{4} d x-2 R_{n}^{2}+T_{n}\right)^{2}<\infty
$$

for almost all $t$. It follows in particular that for almost all $t$ and any $\epsilon>0$ we have for all $n \geqq n_{0}(t, \epsilon)$

$$
\int_{0}^{1}\left|s_{n}(t, x)\right|^{4} d x<(2+\epsilon) R_{n}^{2}
$$

so that 


$$
R_{n}^{-1 / 2} \int_{0}^{1}\left|s_{n}(t, x)\right| d x>(2+\epsilon)^{-1 / 2}
$$

by (3) again. Since $\epsilon>0$ is arbitrary, this proves (2).

3. Remarks. (1) Let us consider the case of $S_{n} / R_{n}^{4}=O(1 / n)$. It will be immediately clear from the argument of $\$ 2$ that we have

$$
\sum_{m=1}^{\infty} I_{m^{2}}<\infty,
$$

so that

$$
\liminf _{m \rightarrow \infty}{R_{m^{2}}^{-1 / 2}}_{0}^{1}\left|s_{m^{2}}(t, x)\right| d x \geqq 2^{-1 / 2}
$$

for almost all $t$.

Suppose now that

$$
R_{(m+1)^{2}} / R_{m^{2}} \rightarrow 1 \text { as } m \rightarrow \infty .
$$

Let $n$ be any integer between $m^{2}$ and $(m+1)^{2}$. Then from the inequality

$$
\begin{aligned}
\left|\int_{0}^{1}\right| s_{n}(t, x)\left|d x-\int_{0}^{1}\right| s_{m^{2}}(t, x)|d x| & \leqq \int_{0}^{1}\left|s_{n}(t, x)-s_{m^{2}}(t, x)\right| d x \\
& \leqq\left(R_{n}-R_{m^{2}}\right)^{1 / 2}
\end{aligned}
$$

it follows that

$$
\begin{aligned}
R_{n}^{-1 / 2} \int_{0}^{1}\left|s_{n}(t, x)\right| d x \geqq & \left(\frac{R_{(m+1)^{2}}}{R_{m^{2}}}\right)^{-1 / 2} R_{m^{2}}^{-1 / 2} \int_{0}^{1}\left|s_{m^{2}}(t, x)\right| d x \\
& -\left(\frac{R_{(m+1)^{2}}}{R_{m^{2}}}-1\right)^{1 / 2} .
\end{aligned}
$$

Hence

$$
\liminf _{n \rightarrow \infty} R_{n}^{-1 / 2} \int_{0}^{1}\left|s_{n}(t, x)\right| d x \geqq 2^{-1 / 2}
$$

for almost all $t$.

As an example we take $c_{k}=1(k=1,2, \cdots)$. We have then $R_{n}=T_{n}=n$, and $S_{n}=O\left(n^{2}\right)$ for any sequence $\left(m_{k}\right)$ of distinct integers. Therefore 


$$
\underset{n \rightarrow \infty}{\liminf n^{-1 / 2}} \int_{0}^{1}\left|\sum_{k=1}^{n} \phi_{k}(t) e\left(m_{k} x\right)\right| d x \geqq 2^{-1 / 2}
$$

almost everywhere in $t$.

(2) It is obvious that our Theorems 1 and 2 have analogues for partial sums $t_{n}(t, x)$ of real trigonometric series

$$
\sum_{k=1}^{\infty} \phi_{k}(t) a_{k} \cos 2 \pi\left(m_{k} x-\alpha_{k}\right)
$$

with real coefficients $a_{k}$ and phases $\alpha_{k}$. Put

$$
P_{n}=\frac{1}{2} \sum_{k=1}^{n} a_{k}^{2}
$$

and

$$
Q_{n}=\sum a_{i}^{2} a_{j}^{2} a_{k}^{2} a_{l}^{2}
$$

where the summation is taken over $1 \leqq i, j, k, l \leqq n$ such that

$$
\pm m_{i} \pm m_{j} \pm m_{k} \pm m_{l}=0 \text {. }
$$

Then, given any $\epsilon>0$, we have for all $t$ but a set of measure less than $\epsilon$ and all $n \geqq 1$

$$
\int_{0}^{1}\left|t_{n}(t, x)\right| d x \geqq C_{6} P_{n}^{1 / 2}
$$

with some constant $C_{\varepsilon}>0$, and, if $\beta>1$ or $\beta=1$ and $P_{(m+1)^{2}} / P_{m}^{2} \rightarrow 1$ as $m \rightarrow \infty$, where $Q_{n} / P_{n}^{4}=O\left(n^{-\beta}\right)$, we have for almost all $t$

$$
\liminf _{n \rightarrow \infty} P_{n}^{-1 / 2} \int_{0}^{1}\left|t_{n}(t, x)\right| d x \geqq 3^{-1 / 2}
$$

The proof of these results is quite similar to that of the results for complex polynomials $s_{n}(t, x)$.

(3) Let $m_{1}, \cdots, m_{n}$ be any set of $n$ distinct integers. S. Chowla has conjectured that

$$
\min _{0 \leqq x<1} \sum_{k=1}^{n} \cos 2 \pi m_{k} x<-C n^{1 / 2}
$$

for some absolute constant $C>0$ (cf. [1]). If this conjecture is true, it is essentially the best possible. 
We can show that, given $n$ distinct integers $m_{1}, \cdots, m_{n}$, there exists always a subset $m_{i_{1}}, \cdots, m_{i_{r}}$ of $m_{1}, \cdots, m_{n}$ for which

$$
\min _{0 \leqq x<1} \sum_{j=1}^{r} \cos 2 \pi m_{i j} x<-\frac{1}{4}\left(\frac{n}{6}\right)^{1 / 2} \text {. }
$$

This is an easy consequence of the fact that

$$
\int_{0}^{1}\left|\sum_{k=1}^{n} \epsilon_{k} \cos 2 \pi m_{k} x\right| d x>\left(\frac{n}{6}\right)^{1 / 2}
$$

for some sequence $\left(\epsilon_{k}\right)$ of \pm 1 , which is a particular case of the results mentioned in (2).

\section{REFERENCES}

1. P. Erdös, Some unsolved problems, Publ. Math. Inst. Hungar. Acad. Sci. 6 Ser. A (1961), 221-254 (especially, pp. 247-248).

2. R. Salem and A. Zygmund, Some properties of trigonometric series whose terms have random signs, Acta Math. 91 (1954), 245-301.

Horkaido University, SAPPoro, Japan 